\title{
Human amniotic membrane in the treatment of MRONJ
}

\author{
Matteo Val ${ }^{1}$, Diletta Trojan, Luca Guarda Nardini², FRANCESCA FAVARETTO, Mirko Ragazzo \\ 1 Azienda ULSS 2 Marca trevigiana \\ 2 University of Padua
}

Funding: The author(s) received no specific funding for this work.

Potential competing interests: The author(s) declared that no potential competing interests exist.

\section{Abstract}

\section{Purpose}

Human amniotic membrane (HAM) has biological properties which are important for the experimental and clinical applications in managing patients of various medical specialties such as oral and maxilla-facial surgery.

The aim was to evaluate the use of HAM as improvement in surgical wound healing and reduction of relapse in patient affected by medication-related osteonecrosis of the jaw (MRONJ).

\section{Materials and Methods}

A prospective cohort of patients with the diagnosis of MRONJ at the unit of Maxillofacial Surgery of the Ca Foncello Hospital in Treviso, Italy was selected for the study from October 2016 and May 2019. The patients underwent surgical treatment with the placement of amniotic membrane patches. Staging of the lesions was performed according to classification of MRONJ set by the Italian Society of oral medicine with Italian Society of maxillofacial surgery. A multivariate logistic regression was conducted to evaluate the effects of surgical therapy on disease resolution.

\section{Results}

27 patients were included in this study (a total of 34 surgical sites) with a mean age of 68.48 years. The sample involved 6 male and 21 female. 16 patients were assuming Zoledronic acid. Antiresorptive/Antiangiogenic agents were being taken by 16 oncological patients (57\%). The median follow-up period was 14,39 months.

Only 2 patients after 30 days since they were surgically treated showed persistent bone exposure. Both patients were retreated with success.

\section{Conclusions}

The use of the amniotic membrane has a learning curve that is fast enough to justify its routine use, in order to improve the predictability of the interventions for the management of MRONJ. In particular, implement the patient's quality of life and reduce the risk of relapse. The work stands as a pilot study in order to further enhance the results obtained, also introducing a control group.

\section{Introduction}

Medication-related osteonecrosis of the jaw (MRONJ) is a serious side-effect of treatment with anti-resorptive medications.MRONJ is much more common in patients receiving antiresorptive/antiangiogenic drugs for cancer-related 
skeletal events than in patients treated for non-malignant diseases. Key factors for the development of MRONJ are the type and dose of antiresorptive/antiangiogenic drug, an history of trauma, dental surgery or dental infection. Trauma induced by poor fitting or even adequate removable dentures can lead to chronic irritation to gingiva and to the underlying alveolar bone and may trigger osteonecrosis.

For cancer patients, treated with antiresorptive drugs (bisphosphonates (BP), Denosumab) and / or anti-angiogenetic (Bevacizumab), the risk osteonecrosis of the jaw (ONJ) varies between $0 \%$ and $6.7 \%$. For patients with osteometabolic pathology, being treated with anti-reabsorbents (BP, Denosumab), the risk of ONJ varies between 0.004\% and 0.2\%.[1, 2] MRONJ presents major repercussions to the health care system. Although the disease process of MRONJ remains largely unknown and poorly understood, the dominant hypothesis for the pathogenesis of this condition is that patients receiving antiresorptive or antiangiogenic therapies exhibit a diminished ability for bone healing, which in turn triggers a cascade of bone necrosis on traumatic insult to the jaws.[3,4] Symptoms and signs of MRONJ range from mild discomfort, erythema, and intraoral bone exposure to pain, swelling, purulence, ulcerations, fistulae, and pathologic fractures. [5] The best treatment practices for the management of patients with MRONJ is largely debated in literature. Different kind of treatments have been proposed, medical treatments such as antimicrobial mouth rinses, systemic antibiotics, hyperbaric oxygen therapy, pentoxifylline, and teriparatide.[6, 7] And also surgical interventions with different degrees of invasiveness: curettage, sequestrectomy, debridement, and surgical resection.[8-11]

Due to the absence of guidelines in the literature for the correct management of ONJ, various supports to improve the prognosis and reduce the risk of relapse have been proposed for surgical treatment such as: PRF, Buccal fat pad flap, recombinant human BMP-2, Human amniotic membrane (HAM) [6, 12-15].

HAM is a tissue obtained by placenta, which promote the wound's healing process due to the high content of growth factors (EGF, FGF, TGF) and tissue metalloprotease inhibitors (TIMP). Furthermore, it has a reduced immunogenicity, connected with the reduced presence of HLA-A, B, C or $\beta 2$ microglobulin antigens. Finally, the anti-inflammatory property of HAM is connected with the capacity to inhibit pro-inflammatory cytokine expression such as IL-1, IL-2, IL-8, IL-10 and IFN-y. This features makes it suitable for any type of transplant without the need to start immunosuppressive therapy. It appears to be promising in facilitating ONJ post-treatment tissue healing as demonstrated by Ragazzo et al.[12] The present study evaluates the use of HAM in the management of MRONJ. The aim is to investigate the healing proprieties and the disease free-survival associated with the use of the amniotic membrane. In particular, if these characteristics are influenced by systemic, local or pharmacological factors.

\section{MATERIALS AND METHODS}

\section{Study design}

This is a prospective unicentric clinical study, with a control arm. The population included patients who had presented with the diagnosis of MRONJ at Unit of Maxillofacial Surgery of the Ca Foncello Hospital in Treviso (Italy) from October 2016 to May 2019. 


\section{Diagnosis of MRONJ}

The diagnosis of osteonecrosis was performed for each patient by clinical and anamnestic evaluation. Preoperative orthopantomography, CT and incisional biopsy of exposed bone, if present, and surrounding mucosa were performed. Staging of the lesions was performed according to SIPMO-SICMF classification of MRONJ [16, 17]

\section{Patient selection}

All male and female patients with diagnosis of MRONJ were recruited in the study. Patients were excluded if:

- They are not assuming or they had never assumed antiresoptive or antiangiogenetic or inibitor of mTOR drugs.

- They previously underwent radiotherapy of the head-neck region

- Their general conditions made it impossible to undergo surgery

Enrolled patients were randomly divided into 2 groups. One group will be treated exclusively with resective surgery (Group A), while the second group will have been treated with resective surgery and with the insertion of HAM patch (Group B).

\section{Surgical Technique}

All patients were made aware of the benefits and possible complications of the surgical procedure.

Prior to surgery, discontinuation of BP was requested at least 1 month before, with the possibility of resuming this treatment once the surgical sites had healed. Patients started antibiotic profilaxis with amoxicillin and clavulanic acid (875 + $125 \mathrm{mg})$ and Metronidazole (500mg), every 8 hours from 7 days before surgery and they will stopped it after another 7 days. Under general anaesthesia, after infiltration of a local anaesthetic (mepivacaine), a mucoperiosteal flap was performed.

Fistulectomy of the hyperplastic mucosa surrounding the exposed bone was carried out. Furthermore, a debridement of hyperplastic-inflammatory tissue and ostectomy of necrotic bone was performed until fresh bleeding from bone was confirmed. Rotary instruments were used to smoothen out all sharp bony margins. HAM had been placed in the bone defect only in Group B and hermetic suture had been performed. All resected tissues were sent to the pathologist to obtain the definitive diagnosis.[12]

\section{HAM processing}

The placenta is usually sourced from donors undergoing caesarean sections and processed shortly after retrieval. The HAM is carefully detached from the chorion and rinsed with sterile saline solution to remove residual blood. The membrane is flattened on a nitrocellulose membrane filter (Merck Millipore), with its stromal/mesenchymal side facing down, in contact with the filter. Afterwards, the HAM is immersed in a cocktail of antibiotics including vancomycin $100 \mu \mathrm{g} / \mathrm{ml}$ (Hospira), meropenem $200 \mu \mathrm{g} / \mathrm{ml}$ (Fresenius Kabi Italia), and gentamicin $200 \mathrm{mg} / \mathrm{ml}$ (Fisiopharma) at $+4^{\circ} \mathrm{C}$ for $24 \mathrm{~h}$ in sterile conditions, validated for human tissues. HAM was cut in $3 \times 3 \mathrm{~cm}^{2}$ patches and cryopreserved. Microbiological analyses are performed at several stages throughout the process and only HAMs without microbial contamination were considered suitable for implants.[18]

\section{Study Variables}

Long-term complications after surgical treatment of $\mathrm{ONJ}$ were the predictor variables. The results of maximum interest of the study were healing of the flap and pain; while inflammation, hematoma, and other complications were considered 
secondary variables. The third category of variables included age, general condition, pharmacological treatment, site, stage and variables that could be related to the outcome.

\section{Data Collection}

Patients' general state of health, medical history, current medications, and clinical and radiographic features of MRONJ were recorded.

Data regarding the transient complications were collected at 1 week postoperatively. Data regarding the long-term complications were evaluated at 3, 6, 12, and 24 months after surgery. Pain perception was assessed by VAS sacale, at rest, during feeding and phonation. These measurements were performed before the intervention, 7 and 30 days after surgery. Ortopantomography was performed at 6 and 12 months postoperatively.

\section{RESULTS}

Twentyseven patients were treated surgically with resection of the necrotic bone of the jaw and placement of HAM patch. One patient died 1 month after surgery due to a complication related to mammal cancer, and was excluded from the study. Details of the individual patients are given in Table 1. Twentyone of the patients were female and six were male. Their mean age was 68.48 years (range 36-89 years). Follow-up of these patients ranged from 6 to 36 months. The ONJ lesions were located in the maxilla in seven patients, in the mandible in 17 patients, and in both jaws in 3 patient. Fiftheen patients had malignant disease and were treated with $\mathrm{BP} /$ anti-reabsorbents/anti-angiogenic drugs administered intravenously; 10 patients with osteoporosis, one with rheumatoid artritis and another patient suffering for algodystrophy were treated with BPs administered orally (except one patient who was assuming risedronic acid administered intravenously). At the time of diagnosis, an infectious process involved the MRONJ site in 19 patients while bone exposure was detectable in 22 patients and pain was referred by 18 patients. From the SIPMO SICMF staging the patients are subdivided: 2 patients have stage 1 a disease, 9 patients with stage 1b, 3 patients suffering from a stage 2a MRONJ, 12 patients were classified stage $2 \mathrm{~b}$ and 2 patients were stage 3.

In the 7-day post-operative period, the percentage of patients who no longer present pain rises to $92.5 \%$ (25 out 27 ). 2 patients at 30 days since surgery showed an unhealed surgical wound, so they were successfully retreated.

At present (November 2019) 4 patients have died. There are 30 surgical sites in the follow-up and no signs of disease recurrence

\section{DISCUSSION}

HAM has numerous properties, first of all it is poorly immunogenic[19] and consequently does not cause transplant rejection in the recipient. Then HAM has anti-inflammatory, anti-angiogenic properties and regulates tissue scarring, promoting the healing process thanks growth factors (EGF, TGF, PDGF, FGF). [19, 20] The expression of these factors, such as the TGF, is preserved even during cryopreservation of the amniotic membrane at $-80^{\circ} \mathrm{C}$.[21] HAM inhibits pro-inflammatory cytokine expression such as IL-1, IL-2, IL-8, IL-10 and IFN-y; [19] while its anti-angiogenetic action is achieved through the production of endostatin and tissue inhibitors of matalloproteinases (TIMP-1,2,3 and 4). [22] The poor immunogenicity was initially thought to be related to the absence of antigens of the major histocompatibility complex HLA-A, B or DR. [23] Subsequent studies have shown that antigen expression HLA class la complex (HLA-A, B 
$\mathrm{C}, \mathrm{DR})$ and $\mathrm{Ib}(\mathrm{HLA}-\mathrm{E}, \mathrm{G})$ is very limited on the epithelial and mesenchymal cell of the membrane. [24]

The anti-microbial impact of AM and amniotic fluid is attributable to the presence of bactricidin, beta-lysin, lysozyme, transferrin and 7-S immunoglobulins in the amniotic fluid. [25]

HAM seems to be able to reduce pain experienced by patients. The adherence of the amnion to the surgical wound and the coverage of nerve endings is the basis of this phenomenon.[26]

This features makes HAM suitable in oral and maxillofacial surgery, where it has been used since 1969 for the management of mucous defects after the resection of malignant or precancerous lesions, due to the closure of oro-antral communication, for guided bone regeneration, post-traumatic orbital surgery and temporomandibular joint surgery. [26, 27] The present work carries on what had already been proposed in a case report[12] of the Authors of this paper. Because the main pharmacologic effect of $\mathrm{BP} /$ anti-riasorbitive/anti-angiogenic drugs is inhibition of osteoclasts and of bone vascularization, HAM is expected to stimulate both soft tissue healing and bone remodeling, thus contributing to the successful treatment of MRONJ.

\section{CONCLUSION}

The results obtained from this study confirmed what had been hypothesized. HAM increases the long-term success compared to medical treatment and surgery alone $[6,10,11,28]$. The outcomes are similar to those obtained with the use of PRF[29, 30]. It has a good ability to reduce pain since the immediate post-surgery (92.5\% of patients have no pain 7 days after surgery).

\section{References}

1. Dodson TB: The Frequency of Medication-related Osteonecrosis of the Jaw and its Associated Risk Factors. Oral and Maxillofacial Surgery Clinics 27:509, 2015

2. Sammut S MN, Lopes V, Ralston S: Epidemiological study of alendronate-related osteonecrosis of the jaw in the southeast of Scotland. Br J Oral Maxillofac Surg. 54:501, 2012

3. Aghaloo T, Hazboun R, Tetradis S: Pathophysiology of Osteonecrosis of the Jaws. Oral and Maxillofacial Surgery Clinics 27:489, 2015

4. Fantasia JE: The Role of Antiangiogenic Therapy in the Development of Osteonecrosis of the Jaw. Oral and Maxillofacial Surgery Clinics 27:547, 2015

5. Ruggiero SL: Diagnosis and Staging of Medication-Related Osteonecrosis of the Jaw. Oral and Maxillofacial Surgery Clinics 27:479, 2015

6. Campisi G, Fedele S, Fusco V, Pizzo G, Di Fede O, Bedogni A: Epidemiology, clinical manifestations, risk reduction and treatment strategies of jaw osteonecrosis in cancer patients exposed to antiresorptive agents. Future Oncol 10:257, 2014

7. Hoff AO, Toth BB, Altundag K, Johnson MM, Warneke CL, Hu M, Nooka A, Sayegh G, Guarneri V, Desrouleaux K, Cui J, Adamus A, Gagel RF, Hortobagyi GN: Frequency and risk factors associated with osteonecrosis of the jaw in cancer 
patients treated with intravenous bisphosphonates. J Bone Miner Res 23:826, 2008

8. Williams WB, O'Ryan F: Management of Medication-Related Osteonecrosis of the Jaw. Oral and Maxillofacial Surgery Clinics 27:517, 2015

9. Nicolatou-Galitis O, Kouri M, Papadopoulou E, Vardas E, Galiti D, Epstein JB, Elad S, Campisi G, Tsoukalas N, Bektas-Kayhan K, Tan W, Body JJ, Migliorati C, Lalla RV: Osteonecrosis of the jaw related to non-antiresorptive medications: a systematic review. Support Care Cancer 27:383, 2019

10. El-Rabbany M, Lam DK, Shah PS, Azarpazhooh A: Comparison of Management Strategies of Patients with MRONJ: A Retrospective Cohort Study. Journal of Oral and Maxillofacial Surgery 76:e25, 2018

11. El-Rabbany M, Lam DK, Shah PS, Azarpazhooh A: Surgical Management of Medication-Related Osteonecrosis of the Jaw Is Associated With Improved Disease Resolution: A Retrospective Cohort Study. Journal of Oral and Maxillofacial Surgery 77:1816, 2019

12. Ragazzo M, Trojan D, Spagnol L, Paolin A, Guarda Nardini L: Use of amniotic membrane in the treatment of patients with BRONJ: two case reports. J Surg Case Rep 2018:rjy073, 2018

13. Berrone M, Florindi FU, Carbone V, Aldiano C, Pentenero M: Stage 3 Medication-Related Osteonecrosis of the Posterior Maxilla: Surgical Treatment Using a Pedicled Buccal Fat Pad Flap: Case Reports. Journal of Oral and Maxillofacial Surgery 73:2082, 2015

14. Maluf G, Caldas RJ, Silva Santos PS: Use of Leukocyte- and Platelet-Rich Fibrin in the Treatment of MedicationRelated Osteonecrosis of the Jaws. Journal of Oral and Maxillofacial Surgery 76:88, 2018

15. Young H, Patel D, Kwok J: Primary Wound Closure Using L-PRF in Patients With Mronj and Osteoradionecrosis a Case Series. Journal of Oral and Maxillofacial Surgery 74:e35, 2016

16. Bedogni A, Fedele S, Bedogni G, Scoletta M, Favia G, Colella G, Agrillo A, Bettini G, Di Fede O, Oteri G, Fusco V, Gabriele M, Ottolenghi L, Valsecchi S, Porter S, Petruzzi M, Arduino P, D'Amato S, Ungari C, Fung Polly PL, Saia G, Campisi G: Staging of osteonecrosis of the jaw requires computed tomography for accurate definition of the extent of bony disease. Br J Oral Maxillofac Surg 52:603, 2014

17. Bedogni A, Fusco V, Agrillo A, Campisi G: Learning from experience. Proposal of a refined definition and staging system for bisphosphonate-related osteonecrosis of the jaw (BRONJ). Oral Dis 18:621, 2012

18. Serafini A, Riello E, Trojan D, Cogliati E, Palu G, Manganelli R, Paolin A: Evaluation of new antibiotic cocktails against contaminating bacteria found in allograft tissues. Cell Tissue Bank 17:619, 2016

19. Umezawa A, Hasegawa A, Inoue M, Tanuma-Takahashi A, Kajiwara K, Makino H, Chikazawa E, Okamoto A: Amnion-derived cells as a reliable resource for next-generation regenerative medicine. Placenta 84:50, 2019

20. Hashim SNM, Yusof MFH, Zahari W, Noordin K, Kannan TP, Hamid SSA, Mokhtar KI, Ahmad A: Angiogenic potential of extracellular matrix of human amniotic membrane. Tissue Eng Regen Med 13:211, 2016

21. Koizumi NJ, Inatomi TJ, Sotozono CJ, Fullwood NJ, Quantock AJ, Kinoshita S: Growth factor mRNA and protein in preserved human amniotic membrane. Curr Eye Res 20:173, 2000

22. Hao Y, Ma DH, Hwang DG, Kim WS, Zhang F: Identification of antiangiogenic and antiinflammatory proteins in human amniotic membrane. Cornea 19:348, 2000

23. Adinolfi M, Akle CA, McColl I, Fensom AH, Tansley L, Connolly P, Hsi BL, Faulk WP, Travers P, Bodmer WF: 
Expression of HLA antigens, beta 2-microglobulin and enzymes by human amniotic epithelial cells. Nature 295:325, 1982 24. Houlihan JM, Biro PA, Harper HM, Jenkinson HJ, Holmes CH: The human amnion is a site of MHC class Ib expression: evidence for the expression of HLA-E and HLA-G. J Immunol 154:5665, 1995

25. Galask RP, Snyder IS: Antimicrobial factors in amniotic fluid. Am J Obstet Gynecol 106:59, 1970

26. Kesting MR, Wolff KD, Nobis CP, Rohleder NH: Amniotic membrane in oral and maxillofacial surgery. Oral Maxillofac Surg 18:153, 2014

27. Guarda-Nardini L, Trojan D, Montagner G, Cogliati E, Bendini M, Manfredini D: Human Amniotic Membrane Positioning in the Surgical Treatment of Temporomandibular Joint Degenerative Disorder. Case Rep Surg 2019:6037191, 2019

28. Lopes RN, Rabelo GD, Rocha AC, Carvalho PAG, Alves FA: Surgical Therapy for Bisphosphonate-Related Osteonecrosis of the Jaw: Six-Year Experience of a Single Institution. Journal of Oral and Maxillofacial Surgery 73:1288, 2015

29. Park J-H, Kim J-W, Kim S-J: Does the Addition of Bone Morphogenetic Protein 2 to Platelet-Rich Fibrin Improve Healing After Treatment for Medication-Related Osteonecrosis of the Jaw? Journal of Oral and Maxillofacial Surgery 75:1176, 2017

30. Norholt SE, Hartlev J: Surgical treatment of osteonecrosis of the jaw with the use of platelet-rich fibrin: a prospective study of 15 patients. Int J Oral Maxillofac Surg 45:1256, 2016 